\title{
MÉMOIRES ORIGINAUX
}

\section{L'évolution de la Parasitologie médicale \\ par Hervé HARANT}

(Montpellier)

\begin{abstract}
Résumé
L'auteur retrace l'orientation de la Parasitologie dans l'enseignement médical français au cours des trente dernières années, en essayant de retrouver la part qui revient à l'enseigneur, au médecin, au naturaliste, au biologiste des Hôpitaux, au chercheur.
\end{abstract}

\section{Summary}

The author outlines the orientation given to Parasitology in French medical formation during the thirty last years. He trys to define which part must be respectively allowed to pedagogy, medicine, natural sciences, medical biology and research.

J'ai eu l'occasion en 1967, lors de la réunion à Montpellier de la Société française de parasitologie, de retracer l'histoire de cinquante ans de Parasitologie de langue française. Aussi bien, je rappelais l'œuvre de nos maîtres, précurseurs et fondateurs de notre discipline.

Plus spécialement aujourd'hui, je souhaiterais esquisser la matière et l'esprit et les moyens d'expression de la Parasitologie «médicale » au cours des trente dernières années; la discipline, comme beaucoup de choses des temps présents, se trouve 
désormais au carrefour de la tradition et du progrès technique dans une zone de bordure entre les sciences dites fondamentales et les spécialités médicales: croisée des chemins en vérité, où il est difficile de sertir les contours, de délimiter les frontières, de consacrer une autonomie au sein de l'actuelle pluridisciplinarité, impliquant une culture polyvalente.

Parmi les parasitologistes des Facultés de Médecine française, ceux qui ont aujourd'hui plus de 60 ans, ont été consacrés dans la carrière à la suite d'un concours d'Agrégation qui comportait une section d'Histoire naturelle et Parasitologie. Dès 1920, ce vocable d'Histoire naturelle était bien désuet; cette science se mourait. Les sièzles précédents, jusqu'au début du $\mathrm{xx}^{\circ}$, avaient apporté suffisamment de précisions dans l'étude de l'ordonnancement du règne vivant, de l'évolution des êtres et de leur premier développement. On pensait désormais réserver à quelques maniaques le loisir de classer des oiseaux empaillés, de ranger en collection des insectes et de confier à des bocaux poussiéreux la dépouille décolorée et amorphe d'un mollusque ou d'un poisson. Par une sorte d'erreur verbale pareille à celles qui, dans le domaine social et politique, servent tout à coup à l'orientation nouvelle du destin des peuples, la Biologie dite générale ne conservait plus son sens total et compréhensif et s'opposait à la vieille «Histoire naturelle». On oubliait d'être naturaliste: la crise «cytologique» nous avait éloigné de l'in vivo au profit de l'in vitro: tout cela, parce qu'on avait omis de replacer l'être vivant dans son environnement. Et cependant, W. Jaeger dans son admirable ouvrage consacré à l'éducation du jeune Grec (Paideia) nous rappelle qu' $\sigma \tau$ ○ọin dans son acception primitive désigna la recherche physique. Le type d'Histoire le plus ancien vit, en effet, le jour en Ionie, au moment où les sciences de la nature faisaient leurs premiers pas. Dès lors, Hecatée de Milet fut le premier à spécialiser son enquête non plus à l'ensemble de la nature mais à un domaine plus particulier, la terre habitée ou oekoumène. Ultérieurement, les historiens, au sens actuel du mot, firent leur une discipline au service des événements humains, de la protection des territoires, des problèmes économiques de survie, de rivalité de coexistence pacifique, etc. L'essentielle «cobiose» aux dépens de laquelle les êtres vivants subsistent dans un instable équilibre retenait à son tour tardivement l'attention des naturalistes.

Bien que le vocable d'écologie fût adopté par Heckel en 1866 et que le terme de biocoenose fût créé par Möbius dès 1877 et malgré les efforts des phytosociologistes du début $\mathrm{du} \mathrm{xx}^{e}$ sièzle, les zoologistes méconnurent trop longtemps que l'étude des groupements en équilibre devaient prendre le pas sur le besoin de catégoriser des cadavres de diverses « espèces» ou prétendues telles.

Il reste, qu'aux environs de 1920, un étudiant en Sorbonne pouvait devenir licencié ès-sciences naturelles dans de brillantes conditions sans jamais observer un animal vivant $\mathrm{ni}$ au laboratoire ni dans la nature, sans jamais entendre prononcer le mot d'écologie ; il était possible aux meilleurs de disserter longuement sur le développement des Echinodermes ou des Bryozoaires - et ce n'est pas moi qui méconnaîtrais le charme de l'étude des animaux marins - mais l'étudiant d'origine citadine n'aurait pas distingué un pinson d'une fauvette, pas plus qu'un épi d'orge d'un épi de blé. 
Il n'est pas étonnant dès lors que d'aucuns n'aient pas conservé de cette formation un souvenir très efficace, au moment cependant où Nicolle avait inventé l'écologie médicale, où le géographe Max Sorre avait enseigné la valeur des complexes pathogènes, où enfin Emile Brumpt avait parcouru le monde avec une « vista » exceptionnelle de naturaliste fondant une épidémiologie dynamique. C'est pourquoi, je me suis cru autorisé plus tard à écrire : l'épidémiologie est l'histoire naturelle des maladies transmissibles. C'est en ce sens seul qu'on pouvait conserver le vocable \& Histoire naturelle qui était limité du temps de nos concours à la connaissance de quelques questions subsidiaires telles les Champignons vénéneux, les Animaux venimeux, etc... Seule en France dans les Facultés de Médecine, la chaire de Montpellier a porté le nom d'Histoire Naturelle et l'on m'accordera dans l'acception que je viens de définir que l'Ecologie médicale, nouvelle manière, doit être retenue dans le cadre de la Parasitologie médicale. Ainsi, le parasitologiste doit être un naturaliste de terrain capable de dépister les comparses du «complexe pathogène » (vecteurs, hôtes intermédiaires, réservoirs de virus) et de noter leurs interactions; en bref, de mettre une écologie médicale au service de l'épidémiologie dynamique. Cet aspect du problème a été envisagé dans quelques Instituts étrangers, à l'O.M.S., peut-être aussi dans notre Corps de Santé militaire d'outre-mer, pour être pris plus tard en considération dans nos Universités.

Personnellement, en exécutant ma modeste mission d'universitaire provincial, je n'ai pas cessé, depuis trente ans, de défendre ce que je considère comme la «singularité » de notre discipline et, pour tout dire, en utilisant une formule à la mode, comme sa « haute technicité».

Il y a un fossé difficile à combler entre le cytobactériologiste et l'homme de terrain qui prend contact délibérément avec le complexe pathogène total des grandes endémies transmissibles. Cela implique, à la dernière étape de nos recrutements, une option singulière à respecter.

Cependant, lorsque fut virtuellement épuisée l'étude morphologique des parasites, alors même qu'on éprouvait la nécessité de mieux connaître leur environnement, le parasite étant un organisme vivant dans un biotope vivant, les problèmes d'interactions d'abord entre le parasite et l'hôte devaient inévitablement se poser ; logiquement les techniques précises de l'immunologie, issues des doctrines pastoriennes classiques, allaient, entre les mains d'expérimentateurs avisés et adroits, porter leurs fruits. Non seulement, on décelait bien des nuances dans la physiopathologie de l'agression parasitaire, mais on apportait au médecin des méthodes de diagnostic d'une singulière valeur dont les applications sont désormais quotidiennes: on sait combien en helminthologie, protistologie, mycologie, des certitudes, prémisses de thérapeutiques actives ont remplacé les probabilités de parasitoses, là où la microscopie se montrait défaillante. En ce domaine, l'apport des écoles françaises de Bordeaux, de Lille et de Lyon, pour ne citer que les pionniers, fut d'importance et riche de conséquences pratiques.

Dès lors, le parasitologiste, déjà capable de préciser un diagnostic microscopique, de connaître et d'adopter les méthodes de culture, devait être aussi un biologiste 
capable de pratiquer les techniques immunologiques et sérologiques appliquées au diagnostic des maladies parasitaires. Ce « modus operandi » doit être incorporé à la vie quotidienne de nos C.H.U. et, en conséquence, il est incontestable qu'une culture première de microbiologiste sensu lato s'avère absolument nécessaire.

Quelle que soit l'importance de ces considérations, elles ne devraient pas nous laisser oublier notre mission de médecin; il fut de mode, il y a quelques années, de redouter la censure des cliniciens. C'est parce que nous ne sommes pas allés à eux, que trop facilement nous avons pu passer pro parte pour beaucoup d'entre eux, pour d'inutiles maniaques s'intéressant seulement aux «petites bêtes ». La parasitologie des Ecoles de Médecine est une spécialité médicale; elle reste avec sa technicité singulière une branche de la vaste pathologie dont elle adopte les moyens et les buts.

Qui de nous n'a été appelé à enseigner au moins l'esquisse clinique, les indications thérapeutiques et prophylactiques des parasitoses. Les vocables de pathologie parasitaire, de pathologie tropicale sont souvent associés au titre de nos enseignements. Qui de nous n'a pas été invité à une consultation de dépistage, ou dans un service hospitalier à formuler un avis autorisé. Le rôle hospitalier du parasitologiste ne saurait se borner à la reconnaissance microscopique d'une quinzaine d'objets ou à la lecture de quelques figures d'électrophorèse ; ce serait le confondre avec un laborantin.

Pour ma part, je regrette vivement dans la systématisation officielle des disciplines médicales, que la Parasitologie ne soit pas considérée au moins comme une discipline mixte; il semble d'ailleurs, qu'il s'agisse là d'un dosage craintif de majorité plus facile dans les décisions des instances supérieures. C'est dommage ! Au risque de voir quelques cliniciens en détresse en mal d'avancement briguer des chaires de Parasitologie, s'est substituée la menace pour l'avenir d'une invasion dans les Facultés de Médecine, de parasitologistes non médecins.

Ainsi, la matière et l'esprit de la Parasitologie médicale essentiellement pluridisciplinaire dans ses exigences et ses manifestations se propose de former des enseigneurs, des biologistes des hôpitaux, et évidemment des chercheurs. De quelles modalités de moyens dispose-t-on dans le cadre actuel des programmes en vigueur dont on sait bien d'ailleurs, qu'ils n'échapperont pas à de multiples et successives modifications?

Notre «présence » est relativement réduite dans la scolarité normale du type école professionnelle, peut-être précisément, comme je l'écrivais plus haut, parce qu'on n'a pas cherché à s'incorporer pro parte dans l'enseignement de la pathologie spéciale et de l'épidémiologie. En fait, au début du $2^{\circ}$ cycle, on s'adresse à des étudiants encore "généralistes indécis» dont le plus grand nombre exercera une médecine de quartier ou de bourgade. Il faut pour eux, résolument et avec bon sens, rejeter une grande partie des classiques descriptions morphologiques abusives. Il est inutile pour un praticien de connaître l'anatomie d'une douve, d'un schistosome, 
d'un tænia, d'un ascaris, d'une tique, sauf les linéaments qui suffisent à justifier leur place dans le système naturel. En revanche, qu'il n'en sache pas moins que son client, quand lui seront présentés un Ascaris ou des anneaux de Tænia fraîchement émis ou des Oxyures dans un pot de chambre.

Il faut en outre avec quelques exemples bien choisis recourir à la notion de cycle pour préciser les voies d'entrée et de sortie des parasites pathogènes et dégager l'importante notion de maladie transmissible différente de la maladie seulement contagieuse; la mise en place d'une efficace prophylaxie en dépend. Je pense, avec les films dont nous disposons actuellement, que l'usage du cinéma est d'un grand secours pour ce genre de démonstrations.

Au surplus, ce qui demeure essentiel pour le futur praticien, c'est l'art de demander un diagnostic et de savoir « interpréter» les résultats. Ce devrait être là le but essentiel des quelques séances de T.P. ou d'exercices dirigés qui restent à notre disposition, quand il est possible de les réaliser.

Demander un diagnostic suppose l'opportunité du moment, la technique précise du prélèvement et de l'envoi au laboratoire compétent, et je me permets d'ajouter la discrétion efficace d'une sollicitation valable: omettre de s'appuyer sur une référence biologique peut être de la sottise, de la négligence ou de l'ignorance ; mais lorsqu'un résultat satisfaisant et suffisant est acquis, c'est du snobisme scientiste, en médecine quotidienne, de solliciter d'autres examens à la pointe du progrès dont la valeur n'est pas toujours confirmée.

Quant à l'interprétation par le praticien de la réponse du laboratoire, d'aucuns trouveront anormal que l'on puisse prononcer ce mot; on n'ignore pas cependant qu'en sérologie un taux revêt une grande importance dans la conduite ultérieure à tenir par le médecin traitant. Chacun sait et, c'est pour le parasitologiste un désagrément quotidien, que la présence d'un kyste d'Entamoeba dysenteriae dans les fèces d'un patient peut ne rien signifier sans l'étude nuancée de l'anamnèse et du contexte clinique; quel dommage que dans notre jargon officiel, le respect de la loi de priorité, nous invite à utiliser le nom d'espèce «dysenteriae », pour nommer une amibe qui dans un grand nombre de cas n'est, n'a pas été et ne sera ni la cause ni le témoin d'une amibose. Que dire enfin des thérapeutiques intempestives, maladroites et souvent offensantes déclenchées par un résultat d'analyse mal interprété.

En bref, dans le déroulement de la scolarité normale, notre rôle est d'enseigner clairement des choses simples, sans cesse replacées dans la pratique journalière au service de la médecine humaine et encore ces choses simples beaucoup les oublieront; un tout petit nombre désirera en savoir davantage. Ainsi jouera la meilleure des sélections dans l'inscription aux diplômes spécialisés.

La préparation aux diplômes de Médecine exotique (alias de Médecine tropicale) doit comporter une orientation encore plus «pratique courante que l'enseignement réservé aux étudiants du $2^{\circ}$ cycle : sans être obnubilé par la nécessité de connaître les syndromes exclusivement tropicaux, on ne négligera pas d'apprendre à penser la médecine de tous les jours dans un cadre anthropologique, ethnologique, folklo- 
rique, climatique nouveau; les notions concernant l'alimentation de l'enfant et de l'adulte, les incidences des pathologies raciales et des habitudes de vivre seront ici encore autrement plus importantes à connaître que la morphologie spécifique du Plasmodium ovale, le modus operandi de la réaction de Vogel-Minning ou de la réaction circum ovale. Et combien appréciera-t-on tous les jours d'avoir été initiés aux techniques de petite chirurgie d'urgence plutôt qu'aux discussions byzantines concernant les parasites des chromoblastomycoses ou de la maladie de Gilchrist; la médecine française consentirait-elle désormais à oublier le bon sens ?

En revanche, le certificat de Parasitologie médicale et technique laisse tout le champ libre aux exigences des techniques les plus modernes, susceptibles, à la pointe du progrès d'étancher la soif des praticiens biologistes.

Quant à l'enseignement préparatoire au Certificat supérieur spécialisé, il sera évidemment le reflet des travaux de l'école, des recherches auxquels les candidats auront été associés, des stages variés auxquels, ils auront pu être invités.

Je pense, pour ma part, que l'actuel diplôme de Biologie humaine (qui n'est le plus souvent «humaine » que de nom) n'échappe pas à la critique; je me garderai bien de les formuler, tant sont mobiles, inadéquates, fugaces, inopportunes les solutions de remplacement éventuelles.

Aussi bien, nous arrivons là aux frontières délicates à sertir de l'enseignement supérieur spécialisé et de la recherche, limites aux intrications floues et inévitables pourtant quand il s'agit de concentrer sur le même homme enseignements, renseignements, contributions aux diagnostics pratiques, recherches et trouvailles, exigeant de lui habileté manuelle, astuce technique, intuition et tenacité, sagesse doctrinale et témérité expérimentale. Je ne me hasarderai pas à épiloguer sur un problème sans doute insoluble, qui dépasse évidemment les instances de la spécialité. Il y en a beaucoup qui ont trouvé sans chercher; il y en a tant qui cherchent et qui ne trouvent pas, ou qui font semblant de trouver. Tel aura perfectionné un appareil de mesure ou une méthode d'enregistrement qui introduira des progrès inestimables, tel autre aura poursuivi une ingénieuse expérimentation pour aboutir à traduire de médiocres résultats en statistiques prolixes et en courbes désagréables à lire; pour un troisième une intuition géniale jaillira d'une simple nuance perçue au lit du malade ou enregistrée sur le terrain.

Les planificateurs prévoient le travail d'équipe: lui seul serait rentable; il n'est pas toujours inventeur. En fait, la presse des temps présents, la tétanisation impatiente, le piétinement des ambitions ne laissent évidemment plus de place à une méditation kantienne sur la découverte et la hiérarchie des valeurs. 